\title{
PEANUT RESPONSE TO POTASSIUM FERTILIZERS UNDER SANDY SALINE SOIL CONDITIONS
}

\author{
A. M. Elbaalawy(1), M. S. Mahrous ${ }^{(2)}$ and A. A. Akl(2) \\ (1) Soil Sci. Dept., Fac of Agric, Menoufia University, Egypt. \\ (2) Soil, Water and Environment Res. Inst., A. R. C, Egypt.
}

Received: Dec. 17, 2017

Accepted: Jan. 18, 2018

\begin{abstract}
ABSTARCT: Two field experiments were carried out during two successive summer seasons of 2016 and 2017 at Gelbana Village east Suez Canal, North Sinai Governorate, Egypt to study the impact of three sources of $K$-fertilization ( $K$-sulphate, $K$-humate and $K$ silicate) at different application rates i.e. 0, 20, 40 and $60 \mathrm{~kg} K_{2} \mathrm{O} / \mathrm{fed}$. on seed yield, yield components, chemical composition and seeds quality of peanut plants (Arachis hypogae L.) variety (Giza6). Also, effect of $K$ fertilization on salt affected soil $\mathrm{pH}, \mathrm{EC}$ and content of some available macro- and micro-nutrients were studied. The experiment was carried out in split plot design with three replicates. Increasing rate of added $K$ resulted in a significant increase of seed yield and its components where the highest values were observed with $K$-humate application and the lowest values were associated with $K$ sulphate treatments. Similarly, significant increases of seeds content of $N, P$ and $K(\%)$, $\mathrm{Fe}, \mathrm{Mn}$ and $\mathrm{Zn}(\mathrm{mg} / \mathrm{kg})$, protein and oil contents (\%) were found with increasing rate of added $K$. There were no significant differences in the found values of the studied trails and parameters between the three sources of $K$ fertilization in the two growing seasons. Both soil $\mathrm{pH}$ and EC $\left(\mathrm{dSm}^{-1}\right)$ were decreased with the incremental addition of $K$, where the obvious diminish occurred in soil fertilized by $K$-humate followed by those received K-sulphate. In addition, soil contents ( $\mathrm{mg} / \mathrm{kg}$ ) of available $\mathrm{N}, \mathrm{P}, \mathrm{K} \mathrm{Fe}, \mathrm{Mn}$ and $\mathrm{Zn}$ were augmented with raising the rate of $K$ fertilization, where the highest contents of these nutrients were found in the soil fertilized by $K$-silicate followed by those observed with soils fertilized by $K$-humate. Results obtained from this research indicate the necessity of $K$ fertilization in salt affected soils to improve its properties, increase soil content of available essential nutrients and their agronomic efficiency.
\end{abstract}

Key words: Sandy saline soils, Peanut productivity and quality, $K$-sulphate, $K$-humate and $K$-silicate.

\section{INTRODUCTION}

Peanut (Arachis hypogaea L.) is considered one of the most important edible oil crops in Egypt, due to its seed's high nutritive value for humans as well as produced cake and green leafy hay for feeding livestock, in addition to the importance of the seed oil for industrial purposes. The main growing ares are located in the north of the country, including the reclaimed desert east and west of the Nile Delta. Peanut seeds are characterized by their high oil content $50 \%$, which is utilized in different industries, besides they contain 26-28\% protein, $20 \%$ carbohydrates and $5 \%$ fiber (Fageria et al., 1997).

Soil is the main factor and medium for agricultural production. There are vast areas of desert soils in Egypt either sandy or calcareous in nature that have been cultivated. These soils are characterized by poor physico-chemical properties, soil water-plant relationships and nutritional status (Awaad et al., 2010). So, fertilizer application to these soils is inevitable for achieving high yields. Moreover, soil salinization is one of the major causes of declining 
agricultural productivity in many arid and semiarid regions of the world.

Potassium, a major plant nutrient required in large quantities, plays important role in many physiological activities of plants and thus helps in achieving optimum crop yield (Grant and Bailey, 1993). Nowadays, potassium fertilizers are applied to almost all commercially grown crops to assure its availability to plants in proper proportions leading to obtain high yield and good quality (Inthichack et al., 2012). Moreover, potassium plays many roles in plants beside its main role as major plant nutrient such as reducing the damage caused by salinity to plants (Bar-Tal et al., 1991), increasing growth, yield and quality of crop yields (Khan et al., 2014) and increasing nutrient use efficiency (Tisdale et al., 2002).

Effect of potassium fertilizer application on different crops and soil properties was reported by some researchers. Aramrak et al. (2007) found that groundnut grown on sandy soils, having low available $\mathrm{K}$ content, responed significantly to potassium fertilizer applied to these soils. Awaad et al. (2010) concluded that application of potassium fertilizers resulted in significant increase in growth parameters of groundnut plants grown in newly reclaimed sandy soils of Egypt. Moreover, potassium application caused increase in soil available content of $\mathrm{N}, \mathrm{P}$ and $\mathrm{K}$ and decrease in soil EC and $\mathrm{pH}$.

Different sources of potassium salts can be used as $K$ fertilizers such as potassium chloride $(\mathrm{KCl})$, potassium sulphate $\left(\mathrm{K}_{2} \mathrm{SO}_{4}\right)$, potassium nitrate $\left(\mathrm{KNO}_{3}\right)$, mono-potassium phosphate $\left(\mathrm{KH}_{2} \mathrm{PO}_{4}\right)$ (Magen, 2004) , potassium silicate $\left(\mathrm{K}_{2} \mathrm{O}_{3} \mathrm{Si}\right)$ (Tokunaga , 1991) and potassium humate. Most of potassium fertilization trials depended on using either potassium sulphate or potassium chloride as a source of $\mathrm{K}$. Studies on using other sources of potassium are still limited.

The purpose of this study is to evaluate the effect of potassium sulphate, potassium humate and potassium silicate as potassium fertilizers on available content of some macro- and micro-nutrient of studied soils and productivity of peanut plants.

\section{MATERIALS AND METHODS}

This study was carried out during two successive summer seasons of 2016 and 2017 , to study the effect of some sources of potassium fertilizers on some soil chemical properties and peanut plants productivity grown on sandy loam saline soil, in Gelbana Village at the NorthWestern Mediterranean Coast of Sinai, between $32^{\circ} 25^{\prime} 59^{\prime \prime} \mathrm{E}$ and $30^{\circ} 38^{\prime} 56^{\prime \prime} \mathrm{N}$, East Suez Canal, North Sinai Governorate, Egypt.

The applied potassium fertilizers are potassium humate, potassium sulphate and potassium silicate at rates of 0,20 , $4060 \mathrm{~kg} \mathrm{~K} \mathrm{~K}_{2} \mathrm{O}$ /fed. $\left(4200 \mathrm{~m}^{2}\right)$. Before planting, surface soil samples $(0-30 \mathrm{~cm})$ were taken from the used soil. Soil samples were air-dried, ground, mixed well, and sieved through a $2 \mathrm{~mm}$ sieve. The samples then were analyzed for determination of some physical and chemical properties and their contents of some available macro- and micronutrients according to the methods described by Cottenie et al. (1982) Page et al. (1986) and Klute (1986). The obtained data were recorded in Table (1). Compost was applied at the rate of 5 $\mathrm{Mg} / \mathrm{fed}$. during soil tillage. Super phosphate $\left(15 \% \mathrm{P}_{2} \mathrm{O}_{5}\right)$ was applied at rate of $200 \mathrm{~kg} / \mathrm{fed}$. Urea $(48 \% \mathrm{~N})$ was applied at rate of $40 \mathrm{~kg} / \mathrm{fed}$. in three equal doses after 21, 45 and 60 days from planting. In addition potassium fertilization in three forms was carried out as soil application in three equal doses at the same time of urea applications. At the same day of 
planting the seeds were inoculated by bio-fertilizers (Rhizobium radiobacter sp strain) using gum media. The used biofertilizer obtained from Agricultural Microbiology Department, Soil, Water and Environment Research Institute. Peanut seeds (Arachis hypogaea L.) variety Giza 6 was obtained from Field Crops Research Institute, Agricultural Research Center, Giza, Egypt. The studied treatments were arranged within the experimental units in a randomized split plot design with three replicates, where potassium sources were distributed in main plots and the sub plots represent application rates of each potassium source.

The area of experimental units was $10.5 \mathrm{~m}^{2}(3.5 \times 3)$. Each unit was divided into seven rows. Two inoculated seeds were planted in each hole at $2 \mathrm{~cm}$ depth and 20 cm distance between the two holes. After 15 days from planting, the plants of each hole were thinned to one plant. Other farming practices were carried out according to the recommendations of
Ministry of Agriculture of Egypt. At harvesting stage, plants of each experimental unit were harvested and separated to straw and pods and weighed separately. Seed samples of each replicates were taken, weighed, oven dried at $70^{\circ} \mathrm{C}$ for $24 \mathrm{hrs}$ and weighed to determine the dry matter yield. A $0.2 \mathrm{~g}$ of oven-dried plant samples was digested using $5 \mathrm{ml}$ of $3: 1 \mathrm{H}_{2} \mathrm{SO}_{4}+$ $\mathrm{HClO}_{4}$ mixture according to the method described by Chapman and Pratt (1961). The digest was diluted into $100 \mathrm{ml}$ using distilled water. Nitrogen, P, K, Fe, Mn and $\mathrm{Zn}$ were determined in the diluted solution according to the method described by Cottenie et al. (1982). Oil and protein contents of peanut seeds were determined according to methods reported in (AOAC 1980). Oil content was obtained by the soxhlet extraction method using diethyl ether. Protein was determined by the Kjeldahl procedure and the factor $\mathrm{N} \times 6.25$ was applied to convert nitrogen in to crud protein.

Table (1): Some physical and chemical properties of the studied soil.

\begin{tabular}{|c|c|c|c|c|c|c|c|c|}
\hline \multicolumn{9}{|c|}{ Physical properties } \\
\hline \multirow{2}{*}{\begin{tabular}{|c}
$\begin{array}{c}\text { Course } \\
\text { sand (\%) }\end{array}$ \\
2.84
\end{tabular}} & $\begin{array}{c}\text { Fine } \\
\text { sand } \\
(\%)\end{array}$ & $\begin{array}{l}\text { Silt } \\
(\%)\end{array}$ & \multicolumn{2}{|c|}{$\begin{array}{l}\text { Clay } \\
(\%)\end{array}$} & Texture & \multicolumn{2}{|c|}{$\begin{array}{l}\text { O.M } \\
(\%)\end{array}$} & $\begin{array}{c}\mathrm{CaCO}_{3} \\
(\%)\end{array}$ \\
\hline & 77.22 & 8.16 & \multicolumn{2}{|c|}{11.78} & Sandy loam & \multicolumn{2}{|c|}{0.54} & 10.37 \\
\hline \multicolumn{9}{|c|}{ Chemical properties } \\
\hline \multirow{2}{*}{$\begin{array}{c}\text { pH } \\
(1: 2.5)\end{array}$} & \multirow{2}{*}{$\begin{array}{c}\mathrm{ECe} \\
(\mathrm{dS} / \mathrm{m})\end{array}$} & \multicolumn{4}{|c|}{ Soluble cations (meq/l) } & \multicolumn{3}{|c|}{ Soluble anions (meq/l) } \\
\hline & & $\mathrm{Ca}^{++}$ & $\mathbf{M g}^{++}$ & $\mathrm{Na}^{+}$ & $\mathrm{K}^{+}$ & $\mathrm{HCO}_{3}^{-}$ & $\mathrm{Cl}^{-}$ & $\mathrm{SO}_{4}^{-}$ \\
\hline 8.10 & 9.53 & 10.39 & 19.08 & 65.00 & 0.83 & 9.53 & 60.00 & 25.77 \\
\hline \multicolumn{9}{|c|}{ Available macro- and micro-nutrients (mg/kg) } \\
\hline \multicolumn{3}{|c|}{ Macro-nutrients } & \multicolumn{6}{|c|}{ Micro-nutrients } \\
\hline $\mathbf{N}$ & $\mathbf{P}$ & K & $\mathrm{Fe}$ & & Mn & Z & & $\mathrm{Cu}$ \\
\hline 33 & 5.68 & 198 & 6.4 & & 3.12 & 0.8 & & 0.59 \\
\hline
\end{tabular}


The obtained data of yield and its components and seed's contents of $\mathrm{N}, \mathrm{P}$ $\mathrm{K}, \mathrm{Fe}, \mathrm{Mn}$ and $\mathrm{Zn}$ were statistically analyzed according Gomez and Gomez (1984) using coStat software. After plant harvesting, surface soil sample $(0-30 \mathrm{~cm})$ of each experimental unit was taken and prepared to determine soil $\mathrm{pH}, \mathrm{EC}\left(\mathrm{dSm}^{-1}\right)$ and its content of available macro ( $N, P$ and $\mathrm{K}$ ) and micro (Fe, $\mathrm{Mn}$ and $\mathrm{Zn}$ ) nutrients according to the methods described by Cottenie et al. (1982) and Page et al. (1982).

\section{RESULTS AND DISCUSSION}

Peanut yield and yield component:

The presented data in Table (2) show yield and yield components of peanut plants planted in saline soils of Egypt affected by different sources of potassium (potassium sulphate, potassium humate and potassium silicate) and application rates (0, 20, 40 and $60 \mathrm{~kg} \mathrm{~K}_{2} \mathrm{O} / \mathrm{fed}$.). These data illustrated that, increasing rate of added $K$ fertilization in the three evaluated sources resulted in a significant increases of the tested yield component. This trend was found in the two growing seasons with a slight increase in all tested yield component in the second growing season than that found in the first season. The latter findings are attributed to the found improvement in the soil chemical properties such as found in decrease of both $\mathrm{pH}$ and EC $\left(\mathrm{dSm}^{-1}\right)$ followed by $\mathrm{K}$ fertilizers additions in the second growing season compared with that may be occurred in the first one. Before that, Tattini et al. (1995) and Jacoby (1999) mentioned that, increasing $K$ in growth medium decreased salinity stress and increased plant growth. With different sources of $K$ fertilizers, Nassar and Abdel-Rahman (2015) and Abdel-All et al. (2017) obtained similar increase effect of $\mathrm{K}$ fertilization on plant growth under different soil conditions.
At the same application rate of the evaluated three sources of $K$ fertilizers, data in Table (2) show a wide response of peanut plants to $K$ sources, where the highest values of the tested yield components i.e., pods yield (Mg/fed.), 100 pods weight (g) 100 seed weight $(g)$ and seeds yield (Mg/fed.) were found in the plants grown in soils fertilized by $\mathrm{K}$ humate followed by those found with treatments of K-silicate. This order was noted in both growing seasons. These findings may be resulted from the effect of added K-source on soil properties and its content of available macro- and micronutrients. Nassar and Abdel-Rahman (2015) elucidated the significant effect of $\mathrm{K}$ humate on potato plants growth and attributed that to many essential plant nutrients presented as main components of $\mathrm{K}$ humate. With $\mathrm{K}$-silicate similar results were obtained by Abdel-All et al. (2017) on onion plants. Recently, ElKoumy et al. (2017) obtained similar results with maize plants fertilized by different $K$ sources under sandy and alluvial soil conditions.

Data in Table (3) show that the rate changes of peanut plants response to the added $\mathrm{K}$ - sources using the parameter namely relative changes "RC" (\%). With the three $K$ sources, all $R C$ values were positive for all yield components under study and increased with the increase rate of added $\mathrm{K}$ fertilizers. According to the found RC values, the evaluated $K$ sources takes the order $\mathrm{K}$-humate $>\mathrm{K}$ silicate> K-sulphate. This trend was found with the four yield components in the two growing seasons. Main observation may be noted from RC (\%) values, with all yield components, RC values in the first season were higher than those found in the second one. This means that, K-fertilization was more effective plant growth in the first season. Values of RC with $\mathrm{K}$ fertilzeres treatments varied from component to another where, 
according to found RC values in the two growing seasons, the tested yield components takes the order: seed yield (Mg/fed.) $>100$ seed weight $(\mathrm{g})>100$ pod weight $(\mathrm{g})>$ pods yield (Mg/fed.). This order means that, $\mathrm{K}$ fertilization have a high positive effect on seeds yield of peanut plants

In addition, data in Table (3) show that, agronomical efficiency "AE" of $K$ fertilizers $(\mathrm{kg} / \mathrm{kg})$ for both pods and seeds yield widely varied from $K$ source to another at different application rates, where highest $A E$ values were found in the plats fertilized by K-humate and lowest values were associated with the treatments of $\mathrm{K}$-sulphate. Also, AE values were decreased with the increase rate of $K$ fertilization, these findings were observed in the two growing seasons. The same data show that, AE values of seeds yield with $K$ fertilization were higher than those of pods yield. These results show the great importance of $K$ fertilization to seeds yield increase. In this respect, Nassar and Abdel-Rahman (2015) Sayed (2016), Abdel-All et al. (2017) and El-Koumy et al. (2017) obtained partially similar results with different plants fertilized by different sources $\mathrm{K}$ fertilizers under conditions of some soils of Egypt.

Table (2): Pod and seed yield of peanut plants grown on sandy saline soils affected by $K$ fertilizers.

\begin{tabular}{|c|c|c|c|c|c|c|c|c|c|}
\hline \multicolumn{2}{|c|}{ Potassium treatments } & \multicolumn{4}{|c|}{2016} & \multicolumn{4}{|c|}{2017} \\
\hline $\begin{array}{c}\mathrm{K} \\
\text { source }\end{array}$ & $\begin{array}{c}\text { Added } \mathrm{K}_{2} \mathrm{O} \\
\left(\mathrm{kg} \mathrm{fed}^{-1} .\right)\end{array}$ & $\begin{array}{l}100 \\
\text { pod } \\
(g)\end{array}$ & $\begin{array}{c}\text { Pod } \\
\text { yield } \\
\left(\mathrm{Mg}^{-1}\right) \\
\left.\text { fed }^{-1}\right)\end{array}$ & $\begin{array}{c}100 \\
\text { seed } \\
\text { (g) }\end{array}$ & $\begin{array}{l}\text { Seed } \\
\text { yield } \\
\left(\mathrm{Mg}^{-}\right. \\
\left.\mathrm{fed}^{-1}\right)\end{array}$ & $\begin{array}{l}100 \\
\text { pod } \\
(\mathrm{g})\end{array}$ & $\begin{array}{l}\text { Pod } \\
\text { yield } \\
\left(\mathrm{Mg}^{-}\right. \\
\left.\text {fed }^{-1}\right)\end{array}$ & $\begin{array}{c}100 \\
\text { seed } \\
\text { (g) }\end{array}$ & $\begin{array}{c}\text { Seed } \\
\text { yield } \\
(\mathrm{Mg} \\
\left.\mathrm{fed}^{-1}\right)\end{array}$ \\
\hline Control & 0 & 233.00 & 1.59 & 75.00 & 1.03 & 238.00 & 1.66 & 78.00 & 1.06 \\
\hline \multirow{4}{*}{ 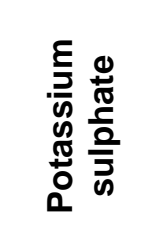 } & 20 & 246.00 & 1.67 & 79.00 & 1.15 & 250.00 & 1.75 & 82.00 & 1.18 \\
\hline & 40 & 250.00 & 1.68 & 82.00 & 1.18 & 253.00 & 1.77 & 84.00 & 1.23 \\
\hline & 60 & 260.00 & 1.70 & 86.00 & 1.22 & 264.00 & 1.82 & 89.00 & 1.25 \\
\hline & Mean & 247.25 & 1.66 & 80.50 & 1.15 & 251.25 & 1.75 & 83.25 & 1.18 \\
\hline \multirow{4}{*}{ 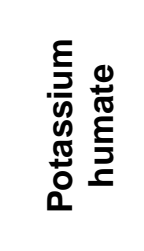 } & 20 & 248.00 & 1.70 & 80.00 & 1.18 & 253.00 & 1.75 & 83.00 & 1.22 \\
\hline & 40 & 259.00 & 1.74 & 86.00 & 1.26 & 261.00 & 1.79 & 88.00 & 1.30 \\
\hline & 60 & 263.00 & 1.78 & 89.00 & 1.30 & 267.00 & 1.86 & 92.00 & 1.33 \\
\hline & Mean & 254.31 & 1.72 & 83.88 & 1.22 & 258.06 & 1.79 & 86.56 & 1.26 \\
\hline \multirow{4}{*}{ 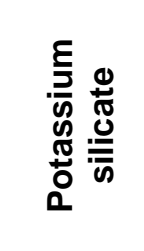 } & 20 & 245.00 & 1.68 & 81.00 & 1.17 & 253.00 & 1.74 & 84.00 & 1.20 \\
\hline & 40 & 259.00 & 1.72 & 87.00 & 1.27 & 264.00 & 1.82 & 89.00 & 1.31 \\
\hline & 60 & 260.00 & 1.74 & 88.00 & 1.31 & 267.00 & 1.85 & 90.00 & 1.35 \\
\hline & Mean & 254.58 & 1.72 & 84.97 & 1.24 & 260.52 & 1.80 & 87.39 & 1.28 \\
\hline \multirow{3}{*}{ LSD } & Sources & NS & NS & NS & NS & NS & NS & NS & NS \\
\hline & Rates & 4.22 & 0.062 & 1.74 & 0.033 & 2.34 & 0.026 & 3.15 & 0.028 \\
\hline & Interaction & NS & NS & NS & NS & 4.68 & NS & NS & NS \\
\hline
\end{tabular}




\begin{tabular}{|c|c|c|c|c|c|c|c|c|c|c|c|c|c|c|c|c|c|c|c|c|c|c|}
\hline \multirow{6}{*}{$\stackrel{乛}{\circ}$} & 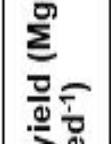 & 山⿸丆𠃋 & & 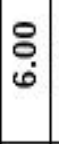 & $\stackrel{\text { ָิ }}{+}$ & $\stackrel{\vec{m}}{\sigma}$ & f & & $\begin{array}{l}\text { ¿े } \\
\infty \\
\infty\end{array}$ & $\begin{array}{l}\stackrel{0}{0} \\
\dot{0}\end{array}$ & 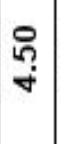 & $\begin{array}{c}\frac{1}{6} \\
\dot{0}\end{array}$ & ' & ì & 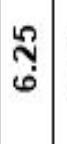 & 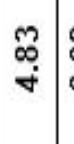 & $\begin{array}{l}m \\
\dot{0} \\
\dot{\omega}\end{array}$ & & 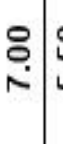 & 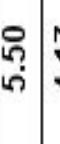 & $\underset{\dot{f}}{-}$ & 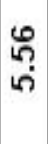 \\
\hline & 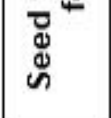 & ఝð & ' & ָึ? & $\begin{array}{l} \pm \\
\dot{0} \\
\stackrel{6}{\sigma}\end{array}$ & \begin{tabular}{l}
\multirow{2}{*}{} \\
$\stackrel{F}{=}$
\end{tabular} & $\begin{array}{l}\stackrel{g}{\circ} \\
\stackrel{1}{\circ}\end{array}$ & ' & $\begin{array}{l}g \\
\stackrel{1}{\circ} \\
\stackrel{\circ}{\circ}\end{array}$ & $\begin{array}{l}\text { ปั: } \\
\text { ป் }\end{array}$ & $\begin{array}{l}\text { ปें } \\
\text { ปे }\end{array}$ & 㐫 & ' & 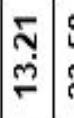 & 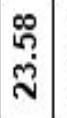 & స̊. & ָั & & 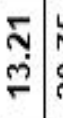 & 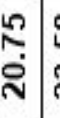 & & $\begin{array}{l}\stackrel{\infty}{\check{\sigma}} \\
\stackrel{\sigma}{\leftarrow}\end{array}$ \\
\hline & 웧ㅇ & (uð & ' & $\frac{m}{i n}$ & : & $\stackrel{\circ}{+}$ & $\begin{array}{l}\hat{\sigma} \\
\infty\end{array}$ & 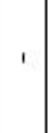 & 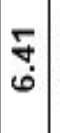 & $\begin{array}{l}\underset{\sim}{\infty} \\
\stackrel{1}{\sim}\end{array}$ & مू & : & ' & : & $\frac{0}{\frac{9}{4}}$ & $\begin{array}{l}\infty \\
\text { ఢి } \\
\sim\end{array}$ & 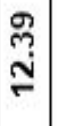 & & $\begin{array}{l}5 \\
\vdots \\
\vdots\end{array}$ & 葛: & $\begin{array}{l}\bar{\phi} \\
\dot{\phi} \\
\dot{\sigma}\end{array}$ & 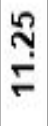 \\
\hline & 풀 & 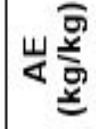 & 1 & 员 & 占 & ஸे & $\bar{m}$ & ' & 予 & సู่ & ले & $\begin{array}{l}9 \\
6 \\
\dot{m}\end{array}$ & ' & 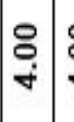 & $\begin{array}{l}\stackrel{8}{\circ} \\
\dot{+}\end{array}$ & $\vec{m}$ & $\begin{array}{l}\underset{N}{N} \\
m\end{array}$ & & 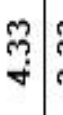 & $\begin{array}{c}m \\
m \\
m\end{array}$ & $\begin{array}{l}\mathscr{1} \\
\dot{m}\end{array}$ & n़े \\
\hline & & પ્ð & 1 & $\begin{array}{l}\text { Fे } \\
\text { เi }\end{array}$ & $\mid \begin{array}{l}0 \\
0 \\
0 \\
0\end{array}$ & \begin{tabular}{|l} 
\\
$\vdots$ \\
$\sigma$
\end{tabular} \mid & 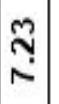 & 1 & $\begin{array}{l}\text { Fै } \\
\text { เึ }\end{array}$ & 范 & 告 & $\begin{array}{c}\stackrel{m}{7} \\
\infty\end{array}$ & ' & $\underset{\sim}{\infty} \underset{\sim}{\infty}$ & $\begin{array}{l} \pm \\
\vdots \\
\sigma \\
0\end{array}$ & $\stackrel{\substack{f \\
\leftarrow}}{=}$ & $\begin{array}{c}m \\
6 \\
\infty \\
\infty\end{array}$ & & $\underset{\sim}{\approx}$ & $\begin{array}{c}m \\
\infty \\
\infty \\
\infty\end{array}$ & 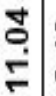 & $\frac{0}{\infty}$ \\
\hline & 음 & ũ & 1 & 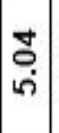 & 总 & 疋 & ヘָ & ' & 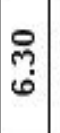 & $\begin{array}{l}\stackrel{0}{0} \\
\stackrel{0}{ }\end{array}$ & 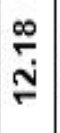 & $\mid$ & ' & 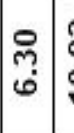 & $\begin{array}{l}\text { N̦ } \\
\stackrel{-}{\circ}\end{array}$ & $\stackrel{\infty}{\stackrel{\infty}{\grave{N}}}$ & $\begin{array}{l}\circ \\
\infty \\
\sigma\end{array}$ & 1 & 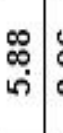 & \begin{tabular}{c|c}
0 \\
$\infty$ \\
$\infty$
\end{tabular} & 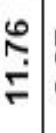 & $\mid \begin{array}{l}\infty \\
\infty \\
\infty\end{array}$ \\
\hline \multirow{6}{*}{$\frac{0}{2}$} & \multirow{2}{*}{ 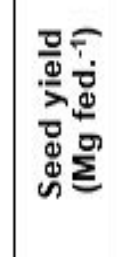 } & 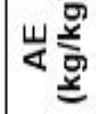 & 1 & $\begin{array}{l}0 \\
0 \\
\dot{0}\end{array}$ & $\begin{array}{l}\stackrel{n}{p} \\
m \\
m\end{array}$ & $\frac{F}{m}$ & $\bar{m}$ & 1 & 号 & $\begin{array}{l}n \\
\text { n. } \\
\text { n. }\end{array}$ & 号 & $\mid \begin{array}{c}\tilde{\sigma} \\
\text { ద่ }\end{array}$ & ' & 인 & @̊ & $\begin{array}{l}\hat{\theta} \\
\dot{\nabla}\end{array}$ & 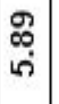 & & $\begin{array}{l}m \\
0 \\
0 \\
\dot{0}\end{array}$ & $\overrightarrow{5}$ & $\underset{f}{F}$ & के \\
\hline & & પ્ð & 1 & 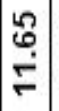 & 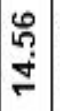 & 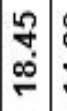 & $\begin{array}{l}\mathscr{g} \\
\infty \\
\dot{j} \\
-\end{array}$ & ' & 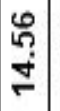 & ָ̃ & $\begin{array}{l}\bar{N} \\
\stackrel{\text { N }}{ }\end{array}$ & $\begin{array}{l}\dot{d} \\
\grave{N}\end{array}$ & ' & 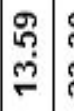 & $\begin{array}{l}\text { ஜे } \\
\text { ஸे }\end{array}$ & $\stackrel{\infty}{\stackrel{\infty}{\sim}}$ & $\begin{array}{l}\stackrel{0}{n} \\
\stackrel{N}{N}\end{array}$ & 1 & \begin{tabular}{l}
\multirow{2}{*}{} \\
$\dot{m}$
\end{tabular} & 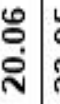 & 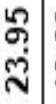 & 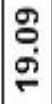 \\
\hline & 웡 & uð & 1 & חृ & m. & $\begin{array}{l}\hat{0} \\
\dot{q} \\
\dot{q}\end{array}$ & 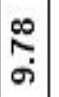 & & $\mid$ & $\begin{array}{l}\overrightarrow{0} \\
\dot{\sigma}\end{array}$ & $\begin{array}{l}\hat{6} \\
\infty \\
\sigma\end{array}$ & $\begin{array}{c}m \\
m \\
m\end{array}$ & . & $\begin{array}{ll}8 & \\
\infty & \\
\infty & \end{array}$ & $\begin{array}{l}\stackrel{0}{0} \\
\dot{0}\end{array}$ & $\stackrel{m}{\stackrel{m}{F}:}$ & $\begin{array}{l}\stackrel{\infty}{c} \\
\stackrel{m}{\rightleftharpoons}\end{array}$ &. & $\begin{array}{l}\hat{\varphi} \\
\dot{0}\end{array} \mid$ & $\begin{array}{c}m \\
m \\
m\end{array}$ & $\begin{array}{l}0 \\
0 \\
\dot{0} \\
\dot{0}\end{array}$ & 总 \\
\hline & 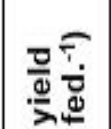 & щ & 1 & 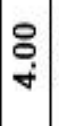 & $\stackrel{\mathfrak{N}}{\text { Na }}$ & 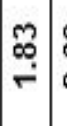 & $\begin{array}{l}0 \\
\stackrel{0}{0} \\
\text { in }\end{array}$ & ' & $\begin{array}{l}0 \\
\text { దी } \\
\text { దी }\end{array}$ & $\begin{array}{l}\stackrel{n}{N} \\
\stackrel{m}{m}\end{array}$ & $\stackrel{F}{\sigma}$ & $\frac{⿱ 亠}{\square}$ & ' & ? & $\underset{\sim}{\mathfrak{n}}$ & గొ & $\underset{\mathcal{F}}{\mathcal{F}}$ & 1 & $\begin{array}{l}\hat{\omega} \\
\dot{\sigma}\end{array}$ & $\begin{array}{l}\infty \\
\stackrel{\infty}{\infty} \\
\dot{m}\end{array}$ & i̊ & ָ̧ \\
\hline & & પ્ð & 1 & 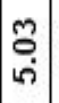 & 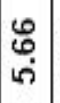 & 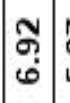 & $\mid$ & ' & 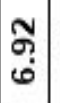 & $\stackrel{m}{q}$ & 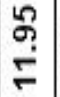 & $\begin{array}{c}\text { gे } \\
\text { के }\end{array}$ & t & $\begin{array}{l}\mathscr{0} \\
\ddot{1}\end{array}$ & $\frac{\infty}{\infty}$ & ఫे & $\begin{array}{l}0 \\
\\
\end{array}$ & 1 & 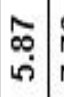 & $\begin{array}{l}0 \\
\\
\end{array}$ & $\begin{array}{l}\text { ma } \\
\sigma\end{array}$ & 量 \\
\hline & 음 & খ̋ & 1 & $\mid$ & ז̊ & 量 & $\frac{10}{\infty}$ & ' & $\begin{array}{l} \\
0 \\
0\end{array}$ & 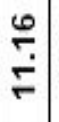 & $\begin{array}{l}\stackrel{\infty}{\infty} \\
\stackrel{0}{\sim} \\
\end{array}$ & $\frac{\varphi}{\check{0}}$ & ' & $\frac{n}{\sin }$ & $\stackrel{\frac{6}{5}}{=}$ & 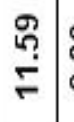 & $\begin{array}{l}\text { ले } \\
\text { के }\end{array}$ & 1 & $\begin{array}{l}\text { Na } \\
\text { ñ }\end{array}$ & 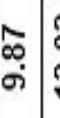 & $\begin{array}{l}\mathfrak{N} \\
\stackrel{\text { ș }}{r}\end{array}$ & సุ. \\
\hline \multirow{2}{*}{ 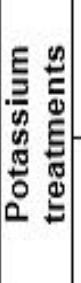 } & 递 & & 0 & iิ & \& & 8 & 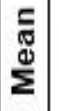 & 0 & సิ & \& & 이 & \begin{tabular}{|l|}
$\mathbf{J}$ \\
$\mathbf{d}$ \\
$\mathbf{\Sigma}$
\end{tabular} & - & సิ & P & $\odot$ & 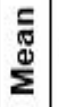 & 0 & ิ & \& & 8 & 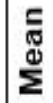 \\
\hline & & 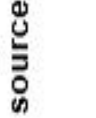 & & $\begin{array}{l}\text { әңе } \\
\text { un! }\end{array}$ & 4dןn & & & & & $\begin{array}{l}\text { eun } \\
\text { SSE }\end{array}$ & & & & & ! & $!_{2}$ & & & & EәW & & \\
\hline
\end{tabular}


Peanut content of macro- concentrations (\%), it may be noted that, nutrients

Seeds of peanut contents of $\mathrm{N}, \mathrm{P}$ and $K(\%)$ were increased with the increase rate of added $\mathrm{K}$ fertilization in the two growing seasons (Table, 4). These findings are due to the effect of added $K$ fertilizers on soil fertility, especially soil content of available $\mathrm{N}, \mathrm{p}$ and $\mathrm{K}$ as mentioned before by El-Koumy et al. (2017). Such increases were found with the three evaluated sources of $K$ fertilizers. Based on the found N, P and K at the same treatment of $\mathrm{K}$ fertilization, peanut seeds contents (\%) of $N, p$ and $K$ in the second growing season were higher than those found in the first one. These increments may be due to the found improvement in soil properties as a result of $K$ fertilization (Abdel-All et al., 2017). Increasing plant content of $N, P$, and $K$ followed by fertilization with different $K$ sources was reported earlier by Belay et al. (2002) and Dong et al. (2010).

Table (4): Effect of $\mathrm{K}$ fertilization on $\mathrm{N}, \mathrm{P}$ and $\mathrm{K}$ content (\%) and their relative changes "RC" (\%) in seeds of peanut plants grown on sandy saline soil.

\begin{tabular}{|c|c|c|c|c|c|c|c|c|c|c|c|c|c|}
\hline \multicolumn{2}{|c|}{$\begin{array}{l}\text { Potassium } \\
\text { treatments }\end{array}$} & \multicolumn{6}{|c|}{2016} & \multicolumn{6}{|c|}{2017} \\
\hline \multirow{2}{*}{$\begin{array}{c}\mathrm{K} \\
\text { source }\end{array}$} & \multirow{2}{*}{$\begin{array}{c}\text { Added } \\
\mathrm{K}_{2} \mathrm{O} \\
\text { (kgfed. }^{-1} \text { ) }\end{array}$} & \multicolumn{2}{|c|}{$N$} & \multicolumn{2}{|c|}{$\mathbf{P}$} & \multicolumn{2}{|c|}{$\mathrm{K}$} & \multicolumn{2}{|c|}{$\mathbf{N}$} & \multicolumn{2}{|c|}{$\mathbf{P}$} & \multicolumn{2}{|c|}{$\mathrm{K}$} \\
\hline & & (\%) & $\begin{array}{l}\text { RC } \\
\text { (\%) }\end{array}$ & (\%) & $\begin{array}{l}\mathrm{RC} \\
\text { (\%) }\end{array}$ & (\%) & $\begin{array}{l}\text { RC } \\
\text { (\%) }\end{array}$ & (\%) & $\begin{array}{l}\mathrm{RC} \\
\text { (\%) }\end{array}$ & (\%) & $\begin{array}{l}\text { RC } \\
(\%)\end{array}$ & (\%) & $\begin{array}{l}\text { RC } \\
\text { (\%) }\end{array}$ \\
\hline \multirow{5}{*}{ 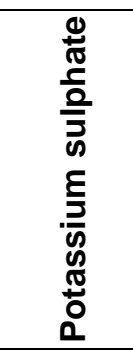 } & 0 & 3.19 & 0.00 & 0.41 & 0.00 & 2.59 & 0.00 & 3.25 & 0.00 & 0.48 & 0.00 & 2.62 & 0.00 \\
\hline & 20 & 3.29 & 3.13 & 0.46 & 12.20 & 2.76 & 6.56 & 3.37 & 3.69 & 0.52 & 8.33 & 2.77 & 5.73 \\
\hline & 40 & 3.34 & 4.70 & 0.51 & 24.39 & 2.91 & 12.36 & 3.44 & 5.85 & 0.58 & 20.83 & 2.94 & 12.21 \\
\hline & 60 & 3.47 & 8.78 & 0.58 & 41.46 & 2.95 & 13.90 & 3.52 & 8.31 & 0.60 & 25.00 & 2.97 & 13.36 \\
\hline & Mean & 3.32 & 5.54 & 0.49 & 26.02 & 2.80 & 10.94 & 3.40 & 5.95 & 0.55 & 18.06 & 2.83 & 10.43 \\
\hline \multirow{5}{*}{ 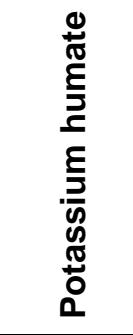 } & 0 & 3.19 & 0.00 & 0.41 & 0.00 & 2.59 & 0.00 & 3.25 & 0.00 & 0.48 & 0.00 & 2.62 & 0.00 \\
\hline & 20 & 3.33 & 4.39 & 0.48 & 17.07 & 2.78 & 7.34 & 3.36 & 3.38 & 0.54 & 12.50 & 2.81 & 7.25 \\
\hline & 40 & 3.46 & 8.46 & 0.54 & 31.71 & 2.88 & 11.20 & 3.42 & 5.23 & 0.59 & 22.92 & 2.91 & 11.07 \\
\hline & 60 & 3.49 & 9.40 & 0.50 & 21.95 & 2.97 & 14.67 & 3.55 & 9.23 & 0.63 & 31.25 & 2.98 & 13.74 \\
\hline & Mean & 3.37 & 7.42 & 0.48 & 23.58 & 2.81 & 11.07 & 3.40 & 5.95 & 0.56 & 22.22 & 2.83 & 10.69 \\
\hline \multirow{5}{*}{ 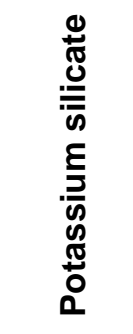 } & 0 & 19 & 0.00 & 0.41 & 0.00 & 2.59 & 00 & 3.25 & 0.00 & 0.48 & 0.00 & 2.62 & 0.00 \\
\hline & 20 & 3.32 & 4.08 & 0.59 & 43.90 & 2.83 & 9.27 & 3.35 & 3.08 & 0.55 & 14.58 & 2.85 & 8.78 \\
\hline & 40 & 3.39 & 6.27 & 0.56 & 36.59 & 2.90 & 11.97 & 3.44 & 5.85 & 0.60 & 25.00 & 2.88 & 9.92 \\
\hline & 60 & 3.49 & 9.40 & 0.52 & 26.83 & 2.98 & 15.06 & 3.56 & 9.54 & 0.63 & 31.25 & 2.92 & 11.45 \\
\hline & Mean & 3.35 & 6.58 & 0.52 & 35.77 & 2.83 & 12 & 3.40 & 6.15 & 0.57 & 23.61 & 2.82 & 10.05 \\
\hline \multirow{3}{*}{$\begin{array}{l}\text { LSD } \\
0.05\end{array}$} & Sources & \multicolumn{2}{|c|}{ NS } & \multicolumn{2}{|c|}{ NS } & \multicolumn{2}{|c|}{ NS } & \multicolumn{2}{|c|}{ NS } & \multicolumn{2}{|c|}{ NS } & \multicolumn{2}{|c|}{ NS } \\
\hline & Rates & \multicolumn{2}{|c|}{0.167} & \multicolumn{2}{|c|}{0.03} & \multicolumn{2}{|c|}{0.012} & \multicolumn{2}{|c|}{0.018} & \multicolumn{2}{|c|}{0.059} & \multicolumn{2}{|c|}{0.036} \\
\hline & Interaction & \multicolumn{2}{|c|}{ NS } & \multicolumn{2}{|c|}{ NS } & \multicolumn{2}{|c|}{ NS } & \multicolumn{2}{|c|}{ NS } & \multicolumn{2}{|c|}{ NS } & & VS \\
\hline
\end{tabular}


Regarding to the agricultural use efficiency of the evaluated three sources of $K$ fertilizers which may be given the terminology of relative changes "RC", data in Table (4) according to N, P and K contents and their relative changes RC (\%) it may observed that, all RC values were positive and increased with the increase rate of added $K$ with no clear trend in the two growing seasons with different sources $K$ sources for $N, P$ and $K$. With all $\mathrm{K}$ fertilization treatments in the two growing seasons, higher RC (\%) values of macronutrients contents were found with $P$ followed by those of $K$. This trend may be resulted from the initial low content of $P$ and high content of available $K$ in the growing medium. The same table shows that, at the same rate of added $K$, the high content of the determined macro-nutrients ( $N, P$ and $K$ ) and their relative changes $R C(\%)$ were found with plants fertilized by K-humate followed by those found in the plants fertilized by $\mathrm{K}$ silicate. These findings may be explained based on the found changes in soil properties and its content of available $\mathrm{N}$, $P$ and $K$ as a result of $K$ additions (EIKoumy et al., 2017). Also, the superior effect K-humate on N, P and K (\%) compared with other sources may be attributed to the presence of these nutrients as essential components of $\mathrm{K}$ humate. Also, both K-humate and Ksilicate improved water status in both plant and soil (Cacco et al., 2000, Jones et al., 2007 and Sayed, 2016). These results are in agreements with those obtained by Kumar et al. (2013), Delfine et al. (2005) and Nassar and AbdelRahman (2015).

\section{Peanut seeds content of micro- nutrient}

Micro-nutrients (Fe, $\mathrm{Mn}$ and $\mathrm{Zn}$ ) content $(\mathrm{mg} / \mathrm{kg})$ in the seeds of peanut plants fertilized by different rates of three
$K$ fertilizers (sulphate, humate and silicate) under sandy loam soil conditions as listed in Table (5) show that, in the two growing seasons and with the three tested $K$ fertilizer sources, increasing rate of added $K$ were associated with an increase in the seeds of peanut contents $(\mathrm{mg} / \mathrm{kg})$ of $\mathrm{Fe}, \mathrm{Mn}$ and $\mathrm{Zn}$. So, all RC values (\%) of the determined micronutrients in peanut seeds were positive in the two growing seasons and become more positive at higher application rate of added $\mathrm{K}$. These findings mainly resulted from the improved conditions of growing medium and increase in micro-nutrients availability followed by $K$ fertilization. Such conclusions were mentioned earlier by Awaad et al. (2010) and Abdel-All et al. (2017).

At the same rate of added $K$, data in Table (5) show a wide variation between the content of the determined micronutrients, where the highest content was found with Fe followed by those recorded with $\mathrm{Mn}$. This trend is in harmony with the used soil content of available micronutrients (Table, 1). Also, at the same rate of added $K$, the found contents of $\mathrm{Fe}, \mathrm{Mn}$ and $\mathrm{Zn}$ in seed of peanut plants in the second growing season were slightly higher than those found in the first season which may be due to improve in soil properties in the second season compared with that found in the first one. On the other hand, RC (\%) values were in the arrangement of $\mathrm{Fe}$ followed by those of $\mathrm{Zn}$ content with different treatments of $\mathrm{K}$ fertilization. In the two growing seasons and at the same rate of added $K$, data in Table (5) showed that high content of $\mathrm{Fe}, \mathrm{Mn}$ and $\mathrm{Zn}$ in peanut seeds were observed in the plants fertilized by K-humate, while lowest values were associated with $\mathrm{K}$-sulphate treatments. So, RC (\%) values of $\mathrm{Fe}, \mathrm{Mn}$ and $\mathrm{Zn}$ content of peanut seeds varied 
from $K$ source to another, where according to theses values the tested $K$ sources takes the order K-humate $>\mathrm{K}$ silicate > K-sulphate. This order was found in the two growing seasons with the determined three nutrients. In this aspect Hashish et al. (2015) and ElKoumy et al. (2017) obtained similar results.

Table (5): Effect of $\mathrm{K}$ fertilization on $\mathrm{Fe}, \mathrm{Mn}$ and $\mathrm{Zn}$ content $\left(\mathrm{mg} \mathrm{kg}^{-1}\right)$ and their relative changes "RC" (\%) in seeds of peanut plants grown on sandy saline soil.

\begin{tabular}{|c|c|c|c|c|c|c|c|c|c|c|c|c|c|}
\hline \multicolumn{2}{|c|}{$\begin{array}{l}\text { Potassium } \\
\text { treatments }\end{array}$} & \multicolumn{6}{|c|}{2016} & \multicolumn{6}{|c|}{2017} \\
\hline \multirow{2}{*}{$\begin{array}{c}\text { K } \\
\text { source }\end{array}$} & \multirow{2}{*}{$\begin{array}{c}\text { Added } \\
\mathrm{K}_{2} \mathrm{O} \\
\left.\text { (kg fed. }^{-1}\right)\end{array}$} & \multicolumn{2}{|c|}{$\mathrm{Fe}$} & \multicolumn{2}{|c|}{ Mn } & \multicolumn{2}{|c|}{$\mathrm{Zn}$} & \multicolumn{2}{|c|}{$\mathrm{Fe}$} & \multicolumn{2}{|c|}{ Mn } & \multicolumn{2}{|c|}{$\mathrm{Zn}$} \\
\hline & & $\begin{array}{l}(\mathrm{mg} \\
\left.\mathrm{kg}^{-1}\right)\end{array}$ & $\begin{array}{l}\text { RC } \\
(\%)\end{array}$ & $\begin{array}{l}(\mathrm{mg} \\
\left.\mathrm{kg}^{-1}\right)\end{array}$ & $\begin{array}{l}\text { RC } \\
(\%)\end{array}$ & $\begin{array}{l}(\mathrm{mg} \\
\left.\mathrm{kg}^{-1}\right)\end{array}$ & $\begin{array}{c}\text { RC } \\
(\%)\end{array}$ & $\begin{array}{l}(\mathrm{mg} \\
\left.\mathrm{kg}^{-1}\right)\end{array}$ & $\begin{array}{l}\text { RC } \\
(\%)\end{array}$ & $\begin{array}{l}(\mathrm{mg} \\
\left.\mathrm{kg}^{-1}\right)\end{array}$ & $\begin{array}{c}\text { RC } \\
(\%)\end{array}$ & $\begin{array}{l}(\mathrm{mg} \\
\left.\mathrm{kg}^{-1}\right)\end{array}$ & $\begin{array}{r}\text { RC } \\
(\%)\end{array}$ \\
\hline \multirow{5}{*}{ 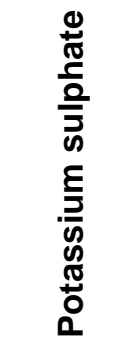 } & 0 & 86.25 & 0.00 & 47 & 0.00 & 36 & 0.00 & 87.12 & 0.00 & 48 & 0.00 & 42 & 0.00 \\
\hline & 20 & 89.30 & 3.54 & 51 & 8.51 & 39 & 8.33 & 89.25 & 2.44 & 59 & 22.92 & 46 & 9.52 \\
\hline & 40 & 91.34 & 5.90 & 55 & 17.02 & 42 & 16.67 & 91.08 & 4.55 & 61 & 27.08 & 47 & 11.90 \\
\hline & 60 & 91.36 & 5.92 & 58 & 23.40 & 44 & 22.22 & 91.11 & 4.58 & 64 & 33.33 & 49 & 16.67 \\
\hline & Mean & 89.56 & 5.12 & 52.75 & 16.31 & 40.25 & 15.74 & 89.64 & 3.86 & 58.00 & 27.78 & 46.00 & 12.70 \\
\hline \multirow{5}{*}{ 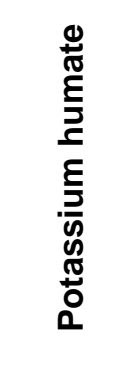 } & 0 & 86.25 & 0.00 & 47 & 0.00 & 36 & 0.00 & 87.12 & 0.00 & 48 & 0.00 & 42 & 0.00 \\
\hline & 20 & 91.36 & 5.92 & 53 & 12.77 & 39 & 8.33 & 91.56 & 5.10 & 62 & 29.17 & 47 & 11.90 \\
\hline & 40 & 92.38 & 7.11 & 58 & 23.40 & 42 & 16.67 & 92.69 & 6.39 & 64 & 33.33 & 49 & 16.67 \\
\hline & 60 & 92.40 & 7.13 & 60 & 27.66 & 45 & 25.00 & 92.72 & 6.43 & 66 & 37.50 & 52 & 23.81 \\
\hline & Mean & 90.60 & 6.72 & 54.5 & 21.28 & 42 & 16.67 & 91.02 & 5.97 & 60.00 & 33.33 & 47.50 & 17.46 \\
\hline \multirow{5}{*}{ 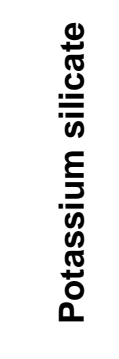 } & 0 & 86.25 & 0.00 & 47 & 0.00 & 36 & 0.00 & 87.12 & 0.00 & 48 & 0.00 & 42 & 0.00 \\
\hline & 20 & 92.35 & 7.07 & 54 & 14.89 & 40 & 11.11 & 92.67 & 6.37 & 63 & 31.25 & 48 & 14.29 \\
\hline & 40 & 92.39 & 7.12 & 59 & 25.53 & 45 & 25.00 & 92.71 & 6.42 & 66 & 37.50 & 50 & 19.05 \\
\hline & 60 & 92.42 & 7.15 & 61 & 29.79 & 49 & 36.11 & 92.76 & 6.47 & 68 & 41.67 & 53 & 26.19 \\
\hline & Mean & 90.85 & 7.11 & 55.25 & 23.40 & 42.5 & 24.07 & 91.32 & 6.42 & 61.25 & 36.81 & 48.25 & 19.84 \\
\hline \multirow{3}{*}{$\begin{array}{l}\text { LSD } \\
0.05\end{array}$} & Sources & \multicolumn{2}{|c|}{0.73} & \multicolumn{2}{|c|}{ NS } & \multicolumn{2}{|c|}{ NS } & \multicolumn{2}{|c|}{0.55} & \multicolumn{2}{|c|}{ NS } & \multicolumn{2}{|c|}{ NS } \\
\hline & Rates & \multicolumn{2}{|c|}{0.83} & \multicolumn{2}{|c|}{5.86} & \multicolumn{2}{|c|}{6.05} & \multicolumn{2}{|c|}{0.85} & \multicolumn{2}{|c|}{0.75} & \multicolumn{2}{|c|}{0.925} \\
\hline & Interaction & \multicolumn{2}{|c|}{ NS } & \multicolumn{2}{|c|}{ NS } & \multicolumn{2}{|c|}{ NS } & \multicolumn{2}{|c|}{ NS } & \multicolumn{2}{|c|}{ NS } & $\mathbf{N}$ & $\mathbf{S}$ \\
\hline
\end{tabular}




\section{Peanut content of protein and oil}

Protein and oil content (\%) of peanut seeds considered the major two parameters which may be used to evaluate seeds quality. Data in Table (6) manifest a significant increase in seeds content of both protein and oil (\%) with increase rate of added $K$. these findings were found in the two growing seasons with the three tested $\mathrm{K}$ fertilizers. Also, oil and protein content (kg/fed) were increased with the increase rate of added $\mathrm{K}$. There are slight increases in both content and yield of oil and protein in the second growing season compared with those found in the first one. These increase resulted from seed high yield and high content of macro- and micronutrients (Tables, 2 to 5). In addition, data in Table (6) show that, all RC values of protein and oil yields affected by rates of added $\mathrm{K}$, were positive and become more positive at higher application rates. Similar increases of protein of wheat plants as a result of $K$ treatment were found by Nassar and Abdel-Rahman (2015). These results are in agreements with those obtained by Anuradha and Sharma (1995), Shahid et al. (1999) and Awaad et al. (2010).

Table (6): Oil and protein content (\%) of peanut plants under sandy saline soil conditions and their relative changes "RC" (\%) affected by $\mathrm{K}$ fertilization.

\begin{tabular}{|c|c|c|c|c|c|c|c|c|c|c|c|c|c|}
\hline \multicolumn{2}{|c|}{ Potassium treatments } & \multicolumn{6}{|c|}{2016} & \multicolumn{6}{|c|}{2017} \\
\hline \multirow[b]{2}{*}{ K source } & \multirow{2}{*}{$\begin{array}{c}\text { Added } \\
\mathrm{K}_{2} \mathrm{O} \\
\text { (kg fed. }^{-1} \text { ) }\end{array}$} & \multicolumn{3}{|c|}{ Oil } & \multicolumn{3}{|c|}{ Protein } & \multicolumn{3}{|c|}{ Oil } & \multicolumn{3}{|c|}{ Protein } \\
\hline & & (\%) & $\begin{array}{c}(\mathrm{Kg} \\
\left.\mathrm{fed}^{-1}\right)\end{array}$ & $\begin{array}{l}\text { RC } \\
\text { (\%) }\end{array}$ & (\%) & $\begin{array}{c}(\mathrm{Kg} \\
\left.\mathrm{fed}^{-1}\right)\end{array}$ & $\begin{array}{l}\mathrm{RC} \\
(\%)\end{array}$ & (\%) & $\begin{array}{c}(\mathrm{Kg} \\
\left.\mathrm{fed}^{-1}\right)\end{array}$ & $\begin{array}{l}\text { RC } \\
\text { (\%) }\end{array}$ & (\%) & $\begin{array}{c}(\mathrm{Kg} \\
\left.\mathrm{fed}^{-1}\right)\end{array}$ & $\begin{array}{l}\text { RC } \\
\text { (\%) }\end{array}$ \\
\hline Control & 0 & 40 & 412.00 & 0.00 & 19.94 & 205.38 & 0.00 & 41 & 434.60 & 0.00 & 20.31 & 215.29 & 0.00 \\
\hline \multirow{4}{*}{$\begin{array}{c}\text { Potassium } \\
\text { sulphate }\end{array}$} & 20 & 42 & 483.00 & 5.00 & 20.56 & 236.44 & 3.11 & 43 & 507.40 & 4.88 & 21.06 & 248.51 & 3.69 \\
\hline & 40 & 43 & 507.40 & 7.50 & 20.87 & 246.27 & 4.66 & 45 & 553.50 & 9.76 & 21.50 & 264.45 & 5.86 \\
\hline & 60 & 44 & 536.80 & 10.00 & 21.69 & 264.62 & 8.78 & 46 & 575.00 & 12.20 & 22.00 & 275.00 & 8.32 \\
\hline & Mean & 42.25 & 484.80 & 5.63 & 20.77 & 238.18 & 4.14 & 43.75 & 517.63 & 6.71 & 21.22 & 250.81 & 4.47 \\
\hline \multirow{5}{*}{$\begin{array}{l}\text { Potassium } \\
\text { humate }\end{array}$} & 0 & 40 & 412.00 & 0.00 & 19.94 & 205.38 & 0.00 & 41 & 434.60 & 0.00 & 20.31 & 215.29 & 0.00 \\
\hline & 20 & 43 & 507.40 & 7.50 & 20.81 & 245.56 & 4.36 & 44 & 536.80 & 7.32 & 21.00 & 256.20 & 3.40 \\
\hline & 40 & 46 & 579.60 & 15.00 & 21.62 & 272.41 & 8.43 & 47 & 611.00 & 14.63 & 21.37 & 277.81 & 5.22 \\
\hline & 60 & 49 & 637.00 & 22.50 & 21.81 & 283.53 & 9.38 & 49 & 651.70 & 19.51 & 22.19 & 295.13 & 9.26 \\
\hline & Mean & 44.5 & 534.00 & 11.25 & 21.05 & 251.72 & 5.54 & 45.25 & 558.53 & 10.37 & 21.22 & 261.11 & 4.47 \\
\hline \multirow{5}{*}{$\begin{array}{l}\text { Potassium } \\
\text { silicate }\end{array}$} & 0 & 40 & 412.00 & 0.00 & 19.94 & 205.38 & 0.00 & 41 & 434.60 & 0.00 & 20.31 & 215.29 & 0.00 \\
\hline & 20 & 42 & 491.40 & 5.00 & 20.75 & 242.78 & 4.06 & 44 & 528.00 & 7.32 & 20.94 & 251.28 & 3.10 \\
\hline & 40 & 46 & 584.20 & 15.00 & 21.87 & 277.75 & 9.68 & 48 & 628.80 & 17.07 & 21.50 & 281.65 & 5.86 \\
\hline & 60 & 48 & 628.80 & 20.00 & 21.81 & 285.71 & 9.38 & 49 & 661.50 & 19.51 & 22.25 & 300.38 & 9.55 \\
\hline & Mean & 44 & 529.10 & 10.00 & 21.09 & 252.90 & 5.78 & 45.5 & 563.23 & 10.98 & 21.25 & 262.15 & 4.63 \\
\hline \multirow{3}{*}{ LSD } & Sources & NS & - & - & NS & - & - & NS & - & - & NS & - & - \\
\hline & Rates & 2.92 & - & - & 0.68 & - & - & 2.85 & - & - & 0.77 & - & - \\
\hline & Interaction & NS & - & - & NS & - & - & NS & - & - & NS & - & - \\
\hline
\end{tabular}


In addition, data in Table (6) elucidate that, at the same rate of added $K$ fertilization in the two growing season, the highest contents (\%) and yields ( $\mathrm{kg} / \mathrm{fed}$ ) of oil and protein were found in the plants fertilized by K-humate followed by those found in plants fertilized by $\mathrm{K}$ sulphate. As mentioned previously, this trend resulted from the improved nutrition status of plants fertilized by $\mathrm{K}$ humate compared with other two sources.

There are no significant difference between the effect of $K$ fertilization sources on the contents of both oil and protein of peanut seeds (Table, 6$)$. These findings means that, $K$ fertilization reduced the harmful effect of soil salinity (Tattini et al., 1995 and Jacoby 1999) and increased quality of peanut yield (Awaad et al., 2010).

\section{Effect of $K$ fertilization on soil properties}

\section{a. Soil pH}

Data in Table (7) denote a slight decrease of sandy loam saline soil pH affected by the $\mathrm{K}$ fertilization, where this decrease effect was increased with raising rate of added $K$. These findings were observed with the three sources of $K$ fertilization in the two growing seasons. The decrease in soil $\mathrm{pH}$ as a result of $K$ fertilization was reported earlier by Abdel-All et al. (2017) and ElKoumy et al. (2017). Based on the mean soil pH affected by the treatments of each $K$ source, these sources take the order: K-sulphate $>\mathrm{K}$-silicate $>\mathrm{K}$-hmate. This order means that, K-humate has a high decrease effect of soil $\mathrm{pH}$ compared with those found with other two sources. This order also may be explained based on $\mathrm{K}$ sources reactions and transformation in the soil.

\section{b. Soil EC (dSm-1) \\ Values of EC $\left(\mathrm{dSm}^{-1}\right)$ of sandy loam}

saline soils fertilized by $K$ in three sources at different application rates, presented in Table (7), show that different $\mathrm{K}$ applications were associated with diminish in soil EC especially at high rates of added $K$. The decline effect of $K$ fertilization on soil EC was observed in the two growing seasons. At the same treatment of $K$ fertilization, soil EC in the first growing season was higher than that found in the second one. These findings were found with the tested three fertilizers. For example EC values of saline soil treated by K-sulphate decreased from 9.12 to 8.31 and from 7.900 to $6.30 \mathrm{dSm}^{-1}$ with the increase rate from 0.0 to $60 \mathrm{~kg} \mathrm{~K} \mathrm{~K}_{2} \mathrm{O}$ fed in the first and second season, respectively. Such this high decrease may be enhanced as a result from other farming practices during two seasons. In this respect Abdel-All et al. (2017) and El-Koumy et al. (2017) obtained similar results.

Regarding to the effect of $K$ fertilization sources on soil EC, data in Table (7) show that, at the same rate of added $K$ and based on the found decrease of soil EC as a result of $K$ fertilization, the tested sources of $K$ fertilization take the order K-silicate (7.57 $\left.\mathrm{dSm}^{-1}\right)>\mathrm{K}$ - humate $\left(7.54 \mathrm{dSm}^{-1}\right)>\mathrm{K}$ sulphate $\left(7.49 \mathrm{dSm}^{-1}\right)$ based on the mean values of $E C$ in the two growing seasons. This arrangement may be ascribed to the added $K$ sources reactions with other soil compounds. Abdel-All et al. (2017) and El-Koumy et al. (2017) obtained similar relations between $\mathrm{K}$ fertilization and Soil EC.

\section{c. Soil content of available macro- nutrients. \\ Sandy loam saline soil content $(\mathrm{mg} / \mathrm{kg})$ of available macro-nutrients affected by $K$ fertilization, represented in Table (7), demonstrate that, increasing rate of added $K$ promoted the soil content of available $N, P$ and $K$. these findings}


A. M. Elbaalawy, et al.,

Table (7): Effect of the studied potassium fertilizers on soil pH, EC and its content of available macro- and micro-nutrients.

\begin{tabular}{|c|c|c|c|c|c|c|c|c|c|}
\hline \multirow{3}{*}{$\begin{array}{l}\text { Potassium } \\
\text { sources }\end{array}$} & \multirow{2}{*}{$\begin{array}{l}\text { Added } \\
\mathrm{K}_{2} \mathrm{O} \\
(\mathrm{Kg} \\
\left.\text { fed }^{-1}\right)\end{array}$} & \multirow[t]{2}{*}{$\begin{array}{c}\text { pH } \\
(1: 2.5)\end{array}$} & \multirow[t]{2}{*}{$\begin{array}{c}E C e \\
\left(d_{S m}^{-1}\right)\end{array}$} & \multicolumn{3}{|c|}{$\begin{array}{c}\text { Available } \\
\text { macronutrients } \\
\left(\mathrm{mgkg}^{-1}\right)\end{array}$} & \multicolumn{3}{|c|}{$\begin{array}{c}\text { Available } \\
\text { micronutrients } \\
\left(\mathrm{mgkg}^{-1}\right)\end{array}$} \\
\hline & & & & $N$ & $\mathbf{P}$ & K & $\mathrm{Fe}$ & $\mathrm{Mn}$ & $\mathrm{Zn}$ \\
\hline & \multicolumn{9}{|c|}{ Season 2016} \\
\hline \multirow{5}{*}{$\begin{array}{l}\text { Potassium } \\
\text { sulphate }\end{array}$} & 0 & 8.08 & 9.12 & 34 & 5.77 & 190 & 6.39 & 3.14 & 0.58 \\
\hline & 20 & 8.04 & 8.18 & 38 & 5.89 & 199 & 6.44 & 3.26 & 0.59 \\
\hline & 40 & 8.00 & 7.97 & 44 & 5.97 & 209 & 6.52 & 3.34 & 0.61 \\
\hline & 60 & 7.97 & 7.83 & 47 & 6.23 & 214 & 6.58 & 3.37 & 0.62 \\
\hline & Mean & 8.02 & 8.28 & 40.75 & 5.97 & 203.00 & 6.48 & 3.28 & 0.60 \\
\hline \multirow{5}{*}{$\begin{array}{l}\text { Potassium } \\
\text { humate }\end{array}$} & 0 & 8.08 & 9.12 & 34 & 5.77 & 190 & 6.39 & 3.14 & 0.58 \\
\hline & 20 & 8.02 & 8.10 & 42 & 5.88 & 206 & 6.68 & 3.17 & 0.56 \\
\hline & 40 & 7.97 & 7.88 & 47 & 6.04 & 215 & 6.72 & 3.24 & 0.59 \\
\hline & 60 & 7.94 & 7.74 & 50 & 6.29 & 218 & 6.75 & 3.32 & 0.59 \\
\hline & Mean & 8.00 & 8.21 & 43.25 & 6.00 & 207.25 & 6.64 & 3.22 & 0.58 \\
\hline \multirow{5}{*}{$\begin{array}{l}\text { Potassium } \\
\text { silicate }\end{array}$} & 0 & 8.08 & 9.12 & 34 & 5.77 & 190 & 6.39 & 3.14 & 0.58 \\
\hline & 20 & 8.06 & 8.38 & 45 & 5.87 & 210 & 6.82 & 3.20 & 0.58 \\
\hline & 40 & 8.02 & 8.14 & 49 & 6.18 & 219 & 6.86 & 3.35 & 0.60 \\
\hline & 60 & 8.01 & 7.18 & 54 & 6.28 & 223 & 6.92 & 3.37 & 0.62 \\
\hline & Mean & 8.04 & 8.38 & 45.50 & 6.03 & 210.50 & 6.75 & 3.27 & 0.59 \\
\hline \multicolumn{10}{|c|}{ Season 2017} \\
\hline \multirow{5}{*}{$\begin{array}{l}\text { Potassium } \\
\text { sulphate }\end{array}$} & 0 & 8.07 & 7.94 & 39 & 5.83 & 196 & 6.45 & 3.18 & 0.59 \\
\hline & 20 & 8.02 & 6.46 & 43 & 5.93 & 203 & 6.53 & 3.34 & 0.61 \\
\hline & 40 & 7.99 & 6.39 & 46 & 5.97 & 211 & 6.56 & 3.38 & 0.61 \\
\hline & 60 & 7.96 & 6.32 & 49 & 5.99 & 216 & 6.60 & 3.41 & 0.62 \\
\hline & Mean & 8.01 & 6.78 & 44.25 & 5.93 & 206.5 & 6.54 & 3.33 & 0.61 \\
\hline \multirow{5}{*}{$\begin{array}{l}\text { Potassium } \\
\text { humate }\end{array}$} & 0 & 8.07 & 7.94 & 39 & 5.83 & 196 & 6.45 & 3.18 & 0.59 \\
\hline & 20 & 8.01 & 6.41 & 45 & 5.97 & 209 & 6.70 & 3.36 & 0.58 \\
\hline & 40 & 7.95 & 6.37 & 51 & 6.04 & 219 & 6.75 & 3.39 & 0.60 \\
\hline & 60 & 7.93 & 6.30 & 63 & 6.08 & 223 & 6.78 & 3.43 & 0.61 \\
\hline & Mean & 7.99 & 6.76 & 49.50 & 5.98 & 211.75 & 6.67 & 3.34 & 0.59 \\
\hline \multirow{5}{*}{$\begin{array}{l}\text { Potassium } \\
\text { silicate }\end{array}$} & 0 & 8.07 & 7.94 & 39 & 5.83 & 196 & 6.45 & 3.18 & 0.59 \\
\hline & 20 & 8.03 & 6.48 & 48 & 5.99 & 213 & 6.84 & 3.39 & 0.59 \\
\hline & 40 & 8.00 & 6.42 & 52 & 6.05 & 222 & 6.89 & 3.43 & 0.61 \\
\hline & 60 & 7.98 & 6.36 & 57 & 6.09 & 226 & 6.95 & 3.46 & 0.62 \\
\hline & Mean & 8.02 & 6.80 & 49.00 & 5.99 & 214.25 & 6.78 & 3.37 & 0.60 \\
\hline
\end{tabular}


were found with the tested three fertilizers in the two growing seasons. There are no wide variations in the soil content of available $\mathrm{N}, \mathrm{P}$ and $\mathrm{K}$ in the two growing seasons. The highest content of the determined available macronutrient under study affected by fertilization was found with $K$ followed by those found with $\mathrm{N}$. In addition, the highest contents of available $\mathrm{K}$ were associated with application of K-humate and the lowest values were found in the experimental units fertilized by K-sulphate. These findings were similar at all application rates of $\mathrm{K}$ in the two growing seasons. These results are in agreement with those obtained by Awaad et al. (2010) and El-Koumy et al. (2017).

\section{d. Soil content of available micro- nutrients}

Contents $(\mathrm{mg} / \mathrm{kg})$ of available $\mathrm{Fe}, \mathrm{Mn}$ and $\mathrm{Zn}$ in the sandy loam saline soil fertilized by three sources of $K$ fertilizers at different rates were determine and the obtained data in Table (7) show that, increasing rate of added $K$ of the three sources (K-humate, K-silicate and Ksulphate) increased soil contents of available $\mathrm{Fe}, \mathrm{Mn}$ and $\mathrm{Zn}$. Similar results were obtained in the two rowing seasons. With all treatments of $K$ fertilization, the highest content was found with $\mathrm{Fe}$ while $\mathrm{Zn}$ recorded the lowest one. In both seasons and according to the soil contents of studied available micronutrients, the tested $K$ sources followed the order: K-silicate > K-humate $>$ K-sulphate. Abdel-All et al. (2017) and El-Koumy et al. (2017) reported similar enhancing effect of $K$ - fertilization on soil content of available $\mathrm{Fe}, \mathrm{Mn}$ and $\mathrm{Zn}$ determined different soil conditions of Egypt.

\section{CONCLUSION}

Based on the obtained data in this study it may be concluded that, soil applications of $K$ fertilizers with the different sources is very important to improve sandy soil productivity of peanut plants as a result of improve in the soil chemical properties and its content of some available macro- and micronutrients especially in the salt affected soil. This means that $\mathrm{K}$ - fertilization increased plant tolerance to salinity stress.

\section{REFERENCES}

Abdel-All, A.E., A. K. Abdelhalim and A. El-Namas (2017). Assessment of deficit irrigation and foliar application of salicylic acid and potassium silicate on performance of drip-irrigated onion crop in sandy soils. Menoufia J. Soil Sci. 2: 29-40.

Anuradha, K. and P.S. Sharma (1995). Effect of moisture stress and applied potassium on yield and biochemical parameters of soybean in Virtisols. J. Oil seeds Res. 12: 275-278.

AOAC (1980). Standard Official Methods of Analysis. 13th Edn., Association of Official Analytical Chemists, Washington, DC., pp: 41-44.

Aramrak, S., J. Chanchareonsook, C. Suwannarat and E. Sarobol (2007). Assessment of multielement extractants for prediction of available potassium in Thai soils. Kasetsart $J$. Natural Sci. 41: 461-466.

Awaad, M. S., A. R. Ahmed and A. A.M. Mohamedin (2010). Effect of different applied of potassium sources on quantity and quality of groundnut crop grown under newly reclaimed sandy soil conditions. Fayoum J. Agric. Res. and Dev. 24: 37-48.

Bar-Tal, A., S. Fergenbaun and D. L. Sparks, (1991). Potassium-salinity interaction in irrigated corn. Irrig. Sci. 12:27-35.

Belay, A., A. Claassens and F. Wehner (2002). Effect of direct nitrogen and potassium and residual phosphorus 
fertilizers on soil chemical properties, microbial components and maize yield under long-term crop rotation. Biol. Fertil. Soils 35:420-427.

Cacco, G., E. Attina, A. Gelsomino and M. Sidari (2000). Effect of nitrate and humic substances of different molecular size on kinetic parameters of nitrate uptake in wheat seedlings. J. Plant Nutr. Soil Sci. 163: 313-320.

Chapman, H.D. and P.F. Pratt (1961). Methods of Analysis for Soils, Plants and Waters. Division of Agricultural Sciences, University of California, Riverside.

Cottenie, A., M. Verloo, L. Kiekens, G. Velgh and R. Camerlynck (1982). Chemical Analysis of Plants and Soils. State Univ. Ghent Belgium.

Delfine, S., E. Tognetti, A. Desiderio and D. Alvino (2005). Effect of foliar application of $\mathrm{N}$ and humic acids on growth and yield of durum wheat. Agron Sustain Dev. 25:183-191

Dong, H., X. Kong, W. Li, W. Tang and D. Zhang (2010). Effects of plant density and nitrogen and potassium fertilization on cotton yield and uptake of major nutrients in two fields with varying fertility. Field Crops Res. 119: 106-113.

El-Koumy, B. Y., Fatma S. El-Shafei, M. M. Shehata and S. S. Ekalawey (2017). Effect of farmyard manure and different sources of $P$ and $K$ on some macronutrients in soil and plant. Menoufia J. Soil Sci. 2:187-200.

Fageria, N.K., V.C. Baligar and C. Jones (1997). Growth and mineral nutrition of field crops 2nd Ed. Marcel Dekker, Inc, New York 1001 k. p. 494.

Gomez, K.A. and A. A. Gomez (1984). Statistical Procedures for Agricultural Research. 2nd Edition, John Wiley and Sons, New York.

Grant, C.A. and L.D. Bailey (1993). Fertility management in canola production. Can. J. Plant Sci., 73: 651670.
Hashish, K.H., F.E.M. El-Quesni and A. M. Mazher (2015). Influence of Potassium humate on growth and chemical constituents of Jatropha curcus L. Int. J. Chem. Tech. Res. 8: 279-283.

Inthichack, P., Y. Nishimura and Y. Fukumoto (2012). Effect of potassium sources and rates on plant growth, mineral absorption, and the incidence of tip burn in cabbage, celery, and lettuce. Hort. Environ. Biotechnol. 53: 135-142.

Jacoby, B (1999). Mechanisms Involved in Salt Tolerance of Plants. In: $M$. Pessarakli (Ed.) Handbook of Plant and Crop Stress, pp. 97-124. New York: Marcel Dekker, Inc.

Jones, C.A., J.S. Jacobsen and A. Mugaas (2007). Effect of low-rate commercial humic acids on phosphorus availability, micronutrient uptake, and spring wheat yield. Comm. Soil Plant Anal. 38: 921-933.

Khan, A. A., M. Sajid, A. Rab, S. Alam and A. Bari (2014). Effect of potassium sources on the growth, yield and fruit quality of tomato cultivars. Sarhad J. Agriculture. 30:442-450.

Klute, A. (1986). Method of Soil Analysis, Part 1, Physical and Mineralogical Methods, Second edition, American Society of Agronomy, Inc and Soil Science Society of America, Inc., Publisher, Madison, Wisconsin USA, $2^{\text {nd }}$ Edition.

Kumar, D., A. P. Singh, P. Raha, A. Rakshit, C.M. Singh and P. Kishor (2013). Potassium Humate: A Potential Soil Conditioner and Plant Growth Promoter. International J. Agriculture, Env. and Biotech. 6:441-446.

Magen, H. (2004). Potassium in Fertigation Systems. International Potash Institute (IPI), 5th Fertigation Training Coarse Boading, AUH, June.

Nassar, K. E. and L. A. Abdel-Rahman (2015). Impact of compost and methods of potassium humate 
application on wheat. Menoufia J.Agr. Res. 40:1377-1385.

Page, A.I., R.A. Miller and D.R. Keene (1982). Methods of Soil Analysis. Part 2: Chemical and Microbiological Properties. $2^{\text {nd }}$ Ed., Amer. Soc. of Agron., Madison, Wisconsin, U.S.A.

Sayed, D. A. (2016). Response of potato plants to potassium and micronutrient fertilization. Menoufia J. Soil Sci. 1:117 $-129$.

Shahid, U., S.K. Bansal, P. Imas and H. Magen (1999). Effect of foliar fertilization of potassium on yield, quality and nutrient uptake of groundnut. J. Plant Nutrition. 22: 1785-1795.
Tattini, M., R. Gucci, M.A. Coradeschi, C. Ponzio and J.D. Everarard (1995). Growth, gas exchange and ion content in Olea europaea plants during salinity and subsequent relief. Physiol. Plantarum 95: 203-210.

Tisdale, S.L., W.L. Nelson, J.D. Beaton and J.L. Havlin (2002). Soil and Fertilizer Potassium. pp. 230-265, In: Soil Fertility and Fertilizers ( $5^{\text {th }}$ Ed.), Prentice Hall of India, New Delhi.

Tokunaga, Y. (1991). Potassium silicate: A slow-release potassium fertilizer. Fertilizer research. 30: 55-59. 
إستجابة الفول السودانى للتسميد البوتاسى تحت ظروف الأراضى الرملية الملحية

أحمد محمد البعلاوى(1) ، محسن صبرى محروس(2) ، أيمن عطفى عقل (2)

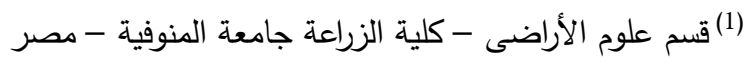

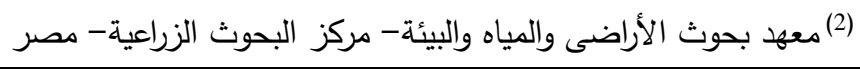

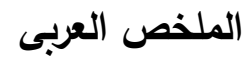

أجريت تجربتين حقليتين خلال الموسم الصيفى لعامى 2016 و 2017 فى قرية جلبانة ; شرق قناة السويس ,

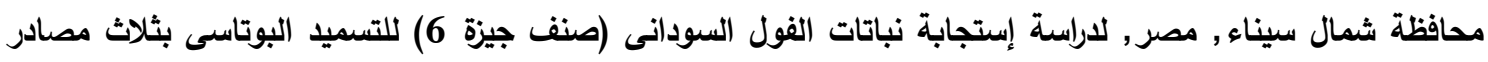

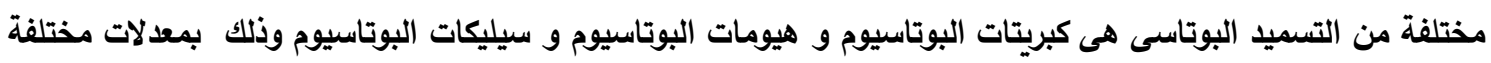

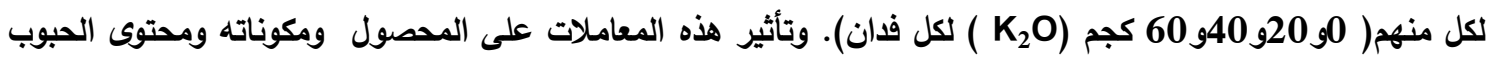

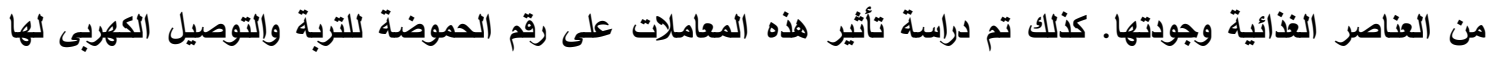
والمحتوى الميسرمن عناصر النيتروجين والفوسفور والبوتاسيوم والحديد والمنجنيز والزنكاتك وصمت التئن التجربة بنظام التطع المنثقة فى ثلاث مكررات. أظهرت النتائج أن زيادة معدلات التسميد البوتاسى أدت إلى زيادة معنوية فى محصول حبوب الفول السودانى وكانت

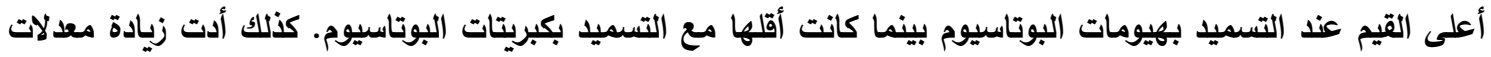

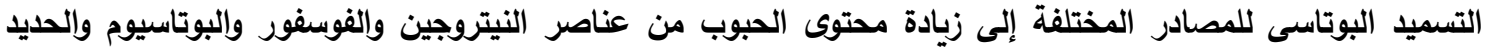

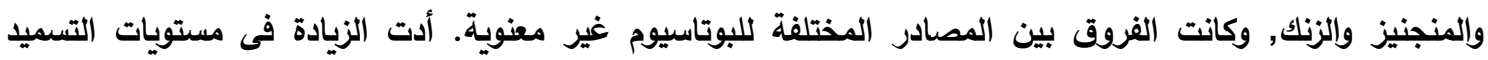

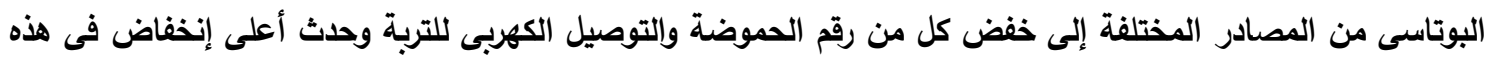

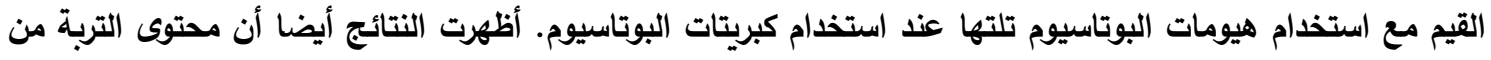

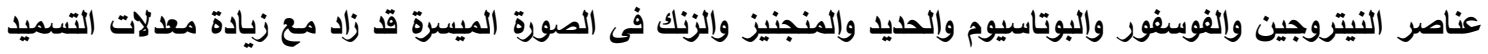

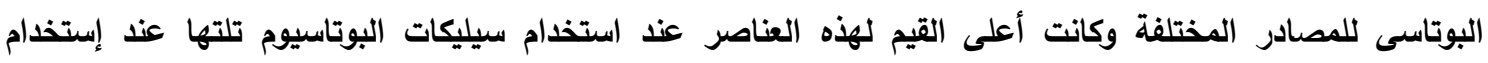

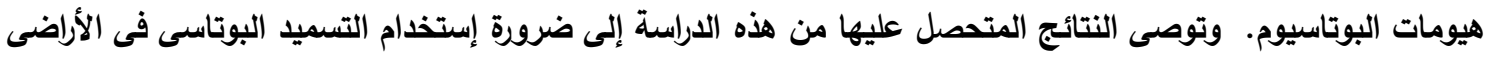
الرملية الملحية لتحسين خواصها وزيادة محتواها من العناصر الذذائية الميسرة و كذلك زيادة كفاءتها لهائا الإنتاجية. 
Peanut response to potassium fertilizers under sandy saline soil conditions 